\title{
On the Hardy class of certain subclass of Bazilevič function
}

\author{
by Mostafa A. NASR (Madras, India)
}

\begin{abstract}
The Hardy classes for a cortain subfamily of Bazilevič functions and their derivative are obtained.
\end{abstract}

Introduction. Let $B_{\alpha}(m)$ be the subclass of normalized Bazilevič functions $f[1]$ defined in the unit disc $E=\{z:|z|<1\}\left(f(0)=0, f^{\prime}(0)=1\right)$, given by

$$
f(z)=\left\{m \int_{0}^{\nu}[g(s)]^{m} p(s) s^{-1} d s\right\}^{L / m},
$$

where $g(z)=z+\sum_{n=2}^{\infty} a_{n} z^{n}$ is a starlike function of order $a, P(z)=1+\sum_{n=1}^{\infty} b_{n} z^{n}$ is analytic function in $E, \operatorname{Re} P(z)>0$ and $m>0$.

Definition. Let $K(\beta, \gamma)$ denote the subclass of the class of closeto-convex function analytic in $E$, such that for $F \in K(\beta, \gamma)$ there exists a starlike function $g$ of order $a$ such that

$$
\left|\arg \frac{z F^{\prime}(z)}{g(z)}\right|<\frac{\beta \pi}{2}
$$

It is well known that $K(0,0)$ is the class of convex function, $K(1,0)$ is the class of close-to-convex functions, and $K(\beta, 0)$ is the subclass of close-to-convex functions of order $\beta$.

The object of this note is to obtain the Hardy classes for $f$ and $f^{\prime}$, where $f \in B_{\mathrm{a}}(m)$.

It is clear that, if $F \in K(\beta, \gamma)$, then

$$
F^{\prime}(z)=\frac{g(z)}{z}\{p(z)\}^{\beta},
$$

where $\operatorname{Re}\{p(z)\}>0$. Denote by $F_{\gamma}(z)$ the function which is obtained from (3) by letting $g .(z)$ be the Koebe function

$$
g_{\gamma}(z)=a z\left(1-z e^{i \tau}\right)^{2 \gamma-2} \quad \text { for some complex } a \text { and real } \tau \text {. }
$$

We need in our proof the following 
LEMMA. If $F \in K(\beta, \gamma)$ and $F$ is not a rotation of $F_{\gamma}$, then there exists $\varepsilon=\varepsilon(F)>0$ such that

$$
F^{\prime} \in H^{1 /[2(1-\gamma)+\not \beta]+8} .
$$

Proof. Since $F \in K(\beta, \gamma)$, then from (3) we have

$$
F^{\prime}(z)=\frac{g(z)}{z} \cdot[p(z)]^{\beta},
$$

where $g(z)$ is starlike of order $\gamma$ and $\operatorname{Re} P(z)>0$. Then from [7], p. 64, it follows that $P(z) \in H^{\lambda}, \forall \lambda, \lambda<1$ and since $g(z) \neq g_{\gamma}(z)$, then from [2], Theorem 5, it follows that

$$
\frac{g(z)}{z} \in H^{1 / 2(1-\gamma)+\delta} \quad \text { for some } \delta=\delta(g)>0 .
$$

Application of Hölder inequalities to $F(z)$ with conjugate indices

$$
p=1+\frac{\beta[1+2 \delta(1-\gamma)]}{2(1-\gamma)} \quad \text { and } \quad q=1+\frac{2(1-\gamma)}{\beta[1+2 \delta(1-\gamma)]}
$$

yields for $z=r e^{i \theta}, 0<r<1$,

$$
\begin{aligned}
& \int_{-\pi}^{\pi}\left|F^{\prime}(z)\right|^{1+2 \delta(1-\gamma) /[2(1-\gamma)+\beta+2 \delta(1-\gamma) \beta]} \\
\leqslant & \left(\int_{-\pi}^{\pi}\left|\frac{g(z)}{z}\right|^{p[1+2 \delta(1-\gamma)] /[2(1-\gamma)+\beta+2 \delta(1-\gamma) \beta]}\right)^{1 / p}\left(\int_{-\pi}^{\pi}|P(z)|^{\frac{\beta q[1+2 \delta(1-\gamma)]_{i}}{2(1-\gamma)+2 \delta(1-\gamma) \beta]}}\right)^{1 / q} .
\end{aligned}
$$

If $\delta$ is sufficiently small, each of the integrals on the right-hand side of the above inequality remains bounded as $r$ tends to 1 . Hence there exists $\varepsilon=\varepsilon(F)>0$ such that

$$
F^{\prime} \in H^{1 /[2(1-\gamma)+\beta]+\varepsilon} \text {. }
$$

Corollary 1. For $0 \leqslant \gamma \leqslant 1 / 2$, from [4], Theorem 33, it follows that there exists $\varepsilon^{\prime}=\varepsilon^{\prime}(F)>0$ such that

$$
F \in H^{1 /[1-2 \gamma+\beta]+\sigma^{\prime}} \text {. }
$$

CoRollary 2. For $\gamma=0$ we can get the result of Theorem 3 [3].

CoRollary 3. For $\gamma=0, \beta=1$ we can get the result of Theorem 6 [2].

Denote by $f_{a}(z)$ the function which is obtain from (1) by letting $g(z)=g_{\mathrm{a}}(z)$.

THEOREM. If $f \in B_{a}(m)$ and $f(z)$ is not a rotation of $f(z)$, then we have

(i) if $0<m<1$, there exists $\varepsilon_{2}=\varepsilon_{2}(f)>0$ such that

$$
f^{\prime} \in H^{1 /[3-2 a m]+\bullet_{1}} \text {, }
$$


(ii) if $m \geqslant 1$, there exists $\varepsilon_{3}=\varepsilon_{3}(f)>0$ such that

$$
f^{\prime} \in H^{1 /[1+2 m(1-a)]+s_{3}} \text {. }
$$

Proof. Since $f \in B_{a}(m)$, from (1) we have

$$
f(z)=\left\{m \int_{0}^{z}\left[\frac{g(s)}{s}\right]^{m} P(s) s^{m-1} d s\right\}^{1 / m} .
$$

Define

$$
F^{\prime}(z)=P^{1 / m}(z)\left(\frac{g(z)}{z}\right)
$$

then from (4) and (5) we have

$$
f(z)=\left\{m \int_{0}^{z}\left[F^{\prime}(z)\right]^{m} s^{m-1} d s\right\}^{1 / m}
$$

and

$$
f^{\prime}(z)=z^{m-1}[f(z)]^{1-m}\left[F^{\prime}(z)\right]^{m} .
$$

Case $1.0<m<1$.

Since $f$ is univalent, then follows from [5], p. 214, that $f(z) \in H^{2}$, $\forall \lambda, \lambda<\frac{1}{2}$ and since $g(z)$ is starlike of order $a$ and is not rotation of $g_{a}(z)$, it follows from the lemma that $F^{\prime}(z) \in H^{m /[2(1-a) m+1]+\varepsilon}$ for some $\varepsilon=\varepsilon(F)>0$. Application of Hölder inequalities to $f(z)$ with conjugate indices

$$
\begin{aligned}
& p=1+\frac{m[1+2 m(1-a)]}{2(1-m)\{m+\varepsilon[1+2 m(1-a)]\}} \\
& q=1+\frac{2(1-m)\{m+\varepsilon[1+2 m(1-a)]\}}{m[1+2 m(1-a)]}
\end{aligned}
$$

yields for $z=r e^{i \theta}(0<r<1)$ that

$$
\begin{aligned}
& \int_{-\pi}^{\pi}\left|\frac{f^{\prime}(z)}{z^{m-1}}\right|^{(m+\varepsilon[1+2 m(1-a)]\} /(m[3-2 m a]+2 \sigma(1-m)[1+2 m(1-a)]\}} \\
& \leqslant\left(\int_{-\pi}^{\pi}|f(z)|^{(1-m) p\{[m+\varepsilon[1+2 m(1-\alpha)]]\} /[m[3-2 m a]+2 e(1-m)[1+2 m(1-\alpha)]]\}}\right)^{1 / p} \times \\
& \quad \times\left(\int_{-\pi}^{\pi}\left|F^{\prime}(z)\right|^{a a\{[m+\varepsilon[1+2 m(1-a]] /[m[3-2 m a]+2 e(1-m)[1+2 m(1-a)]]\}}\right)^{1 / q} .
\end{aligned}
$$

If $\varepsilon$ is sufficiently small, each of the integrals on the right-hand side of the above inequality remains bounded as $r$ tends to 1 , since $[3-2 a m]>1$ 
for $m<1$. Hence there exists $\varepsilon_{1}=\varepsilon_{1}(f)>0$ such that

$$
f^{\prime}(z) \in H^{1 /(3-2 a m)+\varepsilon_{1}} \text {. }
$$

Case 2. $m \geqslant 1$.

It is well known that $\left|f\left(r e^{i \theta}\right)\right| \geqslant r /(1+r)^{2}$ and so from (6), since $m \geqslant 1$ we get

$$
\left|f^{\prime}(z)\right| \leqslant\left(\frac{1}{1+r}\right)^{2(1-m)}\left|F^{\prime}(z)\right|^{m}
$$

and from the lemma, it follows that there exists $\varepsilon_{2}=\varepsilon_{2}(f)>0$ such that

$$
f^{\prime}(z) \in H^{1 /[1+2 m(1-a)]+z_{2}}
$$

Corollary 4. From [4], Theorem 33, under the same conditions of the above theorem, we get, there exists $\varepsilon_{2}=\varepsilon_{3}(f)>0$ such that

$$
f(z) \dot{\epsilon} H^{1 / 2(1-a m)+\varepsilon_{3}} \text {. }
$$

CoRollaRY 5. From the theorem, for $a=0$, we get the result of Theorem 3 [4].

Corollary 6. From Corollary 4 and for $a=0$ we get the result of Theorem 1 [6].

I wish to thank my guide Dr. K. S. Padmanabhan for his encouragement and help in the preparation of this paper.

\section{References}

[1] I. E. B azilevic, On a case of integrability in quadratures of the Lowner-Kufareve equation, Math. J. 37 (79) (1955), p. 471-476.

[2] P. J. E enigenburg and F. R. Keogh, The Hardy class of some univalent functions and their derivatives, Michigan Math. J. 17 (1970), p. 336-346.

[3] P. J. Eenigenburg and S. S. Miller, The $H^{P}$ olasses for $\alpha$-convex function, Proc. Amer. Math. Soc. 38 (1973).

[4] G. H. Hardy and J. E. Littlewood, Some properties of fractional integrals II, Math. Z. 34 (1932), p. 403-439.

[5] J. E. Littlewood, Lectures on the theory of functions, Oxford Univ. Press; Qxford 1944.

[6] S. S. Miller, The Hardy class of Bazilevič function and its derivative, Proc. Amer. Math. Soc. 30 (1971), p. 125-132.

[7] I. I. Priwalow, Raneigenschaften analytischer Funktionen, VEB Deutscher Verl. Wissensch, Berlin 1956. 